\title{
ADENOCARCINOMA DA PRÓSTATA: A ALTERAÇÃO HIPOECOGÊNICA DIFUSA DA PRÓSTATA É UM ACHADO ULTRA-SONOGRÁFICO IMPORTANTE?*
}

\author{
Greice Priscilla Kökeny, Giovanni Guido Cerri², Luciana Mendes de Oliveira Cerri ${ }^{3}$ \\ Nestor de Barros ${ }^{4}$
}

Resumo OBJETIVO: Avaliar se há associação entre a observação de alteração hipoecogênica difusa da próstata, com perda da demarcação entre a zona periférica e a glândula interna, e o diagnóstico de adenocarcinoma de próstata na biópsia prostática transretal. MATERIAIS E MÉTODOS: Avaliamos 143 homens com nível sérico de antígeno prostático específico maior do que $4 \mathrm{ng} / \mathrm{ml}$. Todos os pacientes foram submetidos à ultra-sonografia endorretal e biópsia randomizada da próstata. RESULTADOS: Foi diagnosticado adenocarcinoma de próstata em $\mathbf{3 6 , 4} \%$ dos pacientes. A alteração hipoecogênica difusa da próstata, caracterizada por perda da demarcação entre a zona periférica e a glândula central, foi observada em 22 pacientes e correspondeu ao diagnóstico de adenocarcinoma de próstata em 21 deles (95,4\%). CONCLUSÃO: A alteração hipoecogênica difusa da próstata constituiu um critério de suspeita ultra-sonográfica de adenocarcinoma de próstata altamente significativo, já que em $\mathbf{9 5 , 4} \%$ das próstatas que apresentavam essas características a biópsia foi positiva para adenocarcinoma de próstata

Unitermos: Ultra-sonografia. Ultra-sonografia endorretal. Carcinoma da próstata.

\begin{abstract}
Prostate adenocarcinoma: is transrectal ultrasound diffuse hypoechoic appearance of the prostate an important finding?

PURPOSE: The purpose of this study was to evaluate the relationship between diffuse hypoechoic appearance of the prostate, which is characterized by indistinguishable boundaries between the peripheral zone and the inner gland, and the diagnosis of prostate adenocarcinoma established by transrectal prostate biopsies. MATERIALS AND METHODS: We performed transrectal ultrasonography in 143 male patients with serum prostate specific antigen levels above $4 \mathrm{ng} / \mathrm{ml}$. Randomized biopsies were carried out in all patients. RESULTS: Prostate adenocarcinoma was diagnosed in $36.4 \%$ of the patients. Twenty-one $(95.4 \%)$ out of 22 patients with diffuse hypoechoic appearance of the prostate had positive biopsies for prostate adenocarcinoma. CONCLUSION: Ultrasound diffuse hypoechoic appearance of the prostate is a significant criterion for suspecting adenocarcinoma, since $\mathbf{9 5 . 4} \%$ of the patients exhibiting this ultrasound findings presented positive biopsies. Key words: Ultrasound. Transrectal ultrasound. Prostate carcinoma.
\end{abstract}

\section{INTRODUÇÃO}

Até 1985, a maioria dos estudos relatou o adenocarcinoma da próstata (CAP) como sendo hiperecóico ${ }^{(1-3)}$. O desenvolvimento técnico dos equipamentos de ultra-sonografia (US) ${ }^{(4)}$, aliado à revisão dos conceitos da anatomia da próstata, contribuiu para modificar os critérios da

* Trabalho realizado no Instituto de Radiologia (InRad) do Hospital das Clínicas da Faculdade de Medicina da Universidade de São Paulo (HC-FMUSP), São Paulo, SP.

1. Doutora em Medicina pela FMUSP, Membro Titular do Colégio Brasileiro de Radiologia (CBR).

2. Professor Titular do Departamento de Radiologia da FMUSP, Membro Titular do CBR

3. Médica Assistente do InRad/HC-FMUSP, Membro Titular do CBR.

4. Professor Doutor do Departamento de Radiologia da FMUSP, Membro Titular do CBR

Endereço para correspondência: Dra. Greice Priscilla Kökeny. Rua Cotoxó, 1289, apto. 62, Perdizes. São Paulo, SP, 05021-001. E-mail: nestorbarros@uol.com.br

Aceito para publicação em 21/5/2001 avaliação ultra-sonográfica da glândula prostática $^{(2)}$, e como consequiência houve uma mudança deste conceito de padrão hiperecóico do CAP.

Atualmente, há um consenso de que os CAPs da zona periférica são hipoecóicos na maioria das vezes em que os pacientes são examinados com transdutores de alta freqüência ${ }^{(5)}$, tendo sido relatadas porcentagens de até $70 \%$ em que os CAPs têm esta apresentação ultra-sonográfica ${ }^{(3,6-8)}$. Apesar de o aspecto hipoecóico ser o mais comum, não há uma apresentação patognomônica ultra-sonográfica do $\mathrm{CAP}^{(9)}$.

Os CAPs também podem ser isoecóicos e, portanto, indistinguíveis da próstata normal em cerca de $24 \%$ a $39 \%$ das vezes $^{(\mathbf{1 0 , 1 1 )} \text {. }}$

Além dos aspectos iso, hipo e hiperecóico que o CAP pode apresentar na ultra-sonografia endorretal (USER), devemos estar atentos para avaliar se há perda da demarcação entre a glândula interna e a periférica ${ }^{(\mathbf{1 2})}$.

O objetivo deste trabalho foi avaliar se há associação entre a observação de alteração hipoecogênica difusa da próstata, com perda da demarcação entre a zona periférica e a glândula interna, e o diagnóstico de CAP na biópsia prostática transretal.

\section{MATERIAIS E MÉTODOS}

Foram estudados 143 pacientes do sexo masculino que apresentavam níveis séricos de antígeno prostático específico (PSA) maiores do que $4 \mathrm{ng} / \mathrm{ml}$, dosados por meio do método imunoenzimático, mediante consentimento prévio desses pacientes. Este estudo foi submetido à 
avaliação e aprovado pelo Comitê de Ética da Faculdade de Medicina da Universidade de São Paulo. Não foram incluídos na casuística pacientes que tivessem sido submetidos previamente a cirurgia e/ou radioterapia da próstata. A idade dos pacientes variou de 40 a 88 anos (média de 64 anos).

Estes pacientes fazem parte de uma casuística já apresentada em trabalho publicado anteriormente ${ }^{(13)}$.

Todos os pacientes foram submetidos a USER e biópsia da próstata, por via transretal, após antibioticoterapia profilática (quatro comprimidos de ciprofloxacim $500 \mathrm{mg}$, via oral, de $12 \mathrm{em} 12$ horas, esquema iniciado duas horas antes do início dos procedimentos) e limpeza da ampola retal por intermédio do "fleet-enema".

As biópsias foram realizadas subseqüentemente à avaliação ultra-sonográfica da próstata, nos seus planos longitudinal e transversal, em uma mesma oportunidade, por um dos autores ou sob sua supervisão. O equipamento ultra-sonográfico utilizado foi o Tosbee (Toshiba, Japão), com transdutor biplano de alta frequiência do tipo "end-fire" $(6,0 \mathrm{MHz}$ linear / 7,5 MHz-convexo).

Consideramos alteração hipoecogênica difusa da próstata quando esta apresentava-se difusamente heterogênea e hipoecogênica, com perda da demarca- ção entre a zona periférica e a glândula interna (Figura 1).

Todos os pacientes foram submetidos a biópsias randomizadas através da zona periférica nos sextantes da próstata, identificados como base, médio e ápice, à direita e à esquerda, técnica primeiramente descrita por Hodge et al. ${ }^{(14)}$.

Os fragmentos retirados foram depositados em frascos contendo formol a $6 \%$ e etiquetados, indicando-se as regiões da próstata das quais pertenciam.

Subseqüentemente, foram avaliadas, estatisticamente, as correlações entre os resultados da USER e os da biópsia da próstata. $\mathrm{O}$ teste do qui-quadrado $\left(\chi^{2}\right)$ foi utilizado para avaliar a associação entre a observação de alteração hipoecogênica difusa da próstata e os resultados da biópsia. Adotou-se o nível de significância de $0,05(\alpha=5 \%)$. Níveis descritivos (p) inferiores a este valor foram considerados significantes.

\section{RESULTADOS}

Dentre os 143 pacientes estudados, foi diagnosticado CAP em $36,4 \%$ deles (52/143).

A alteração hipoecogênica difusa da próstata foi observada em 22 dos 143 pacientes estudados, e correspondeu ao diagnóstico de CAP em 95,4\% deles (21/ 22) (Figura 2).
A associação entre a observação de alteração hipoecogênica difusa na US e a positividade de CAP na biópsia foi estatisticamente significante $\left(\chi^{2}=16,82 ; p\right.$ $<0,0001)$.

\section{DISCUSSÃO}

De acordo com a literatura consultada, podemos verificar que há consenso com relação à investigação de CAP em pacientes que apresentam níveis séricos de PSA aumentados ${ }^{(15-17)}$.

Em nosso estudo os pacientes foram submetidos à USER e à biópsia da próstata, por apresentarem níveis séricos de PSA maiores do que $4 \mathrm{ng} / \mathrm{ml}$. No total, detectamos CAP em 36,4\% deles.

A biópsia transretal da próstata tem sido apontada como a melhor técnica para a detecção de CAP nos pacientes com níveis séricos elevados de PSA ${ }^{(18,19)}$. No entanto, as medidas estatísticas da USER, com valores bastante variáveis de sensibilidade e especificidade ${ }^{(20)}$, levaram à tentativa de melhoria da deteç̧ão do CAP por meio deste método, investigando-se outras técnicas associadas, como é o caso do Doppler ${ }^{(21,22)}$ e da utilização dos meios de contraste na $\mathrm{US}^{(23)}$.

Recentemente, a tomografia computadorizada helicoidal da pelve também foi avaliada como método de detecção do $\mathrm{CAP}^{(24)}$. A ressonância magnética tem

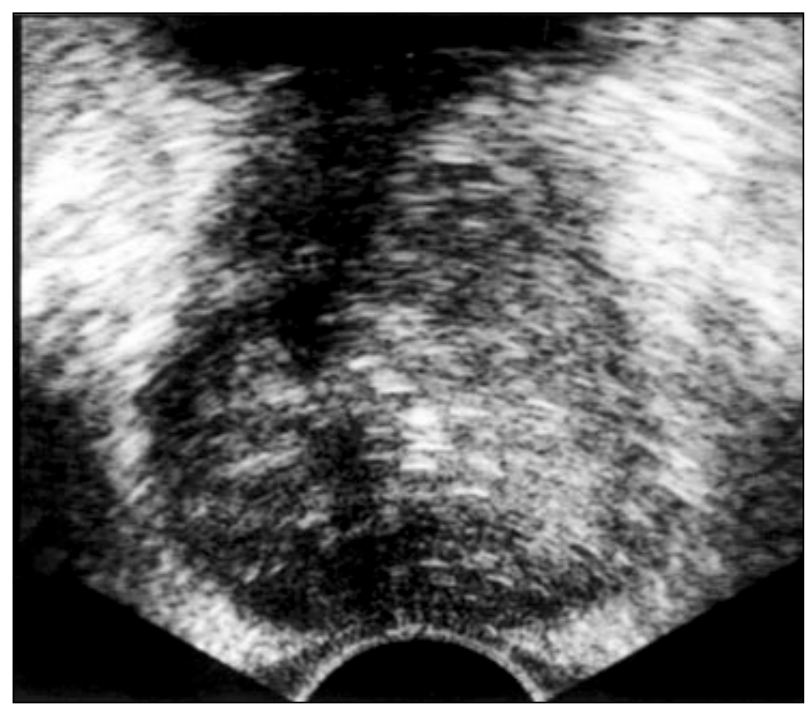

A

Figura 1. Cortes transversais da próstata. Próstata de aspecto difusamente heterogêneo e hipoecogênico, caracterizada por perda da demarcação entre a zona periférica e a glândula interna. 0 resultado da biópsia foi adenocarcinoma.

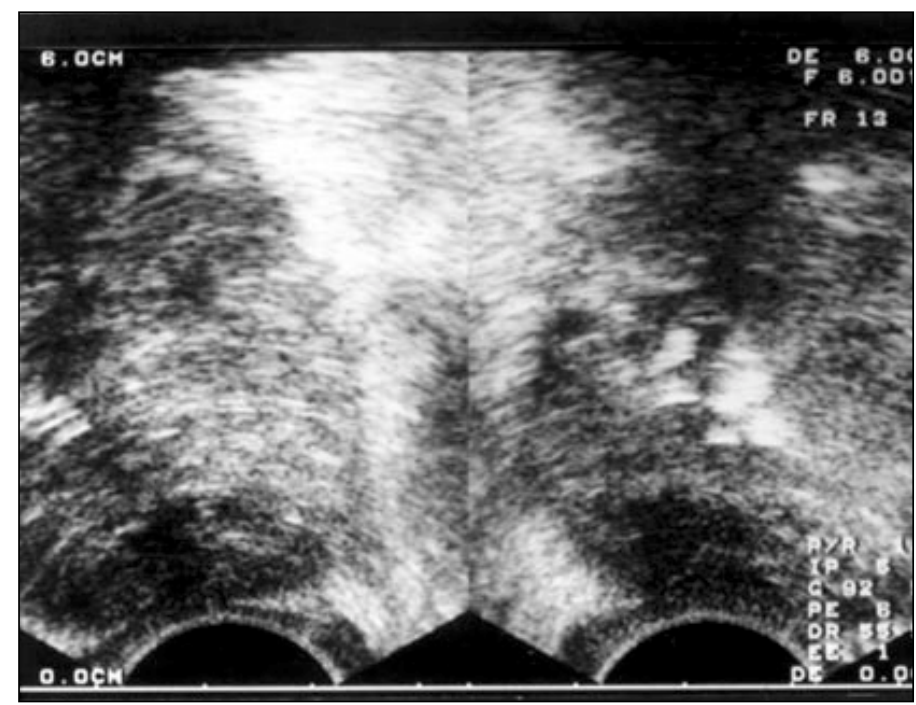

B 


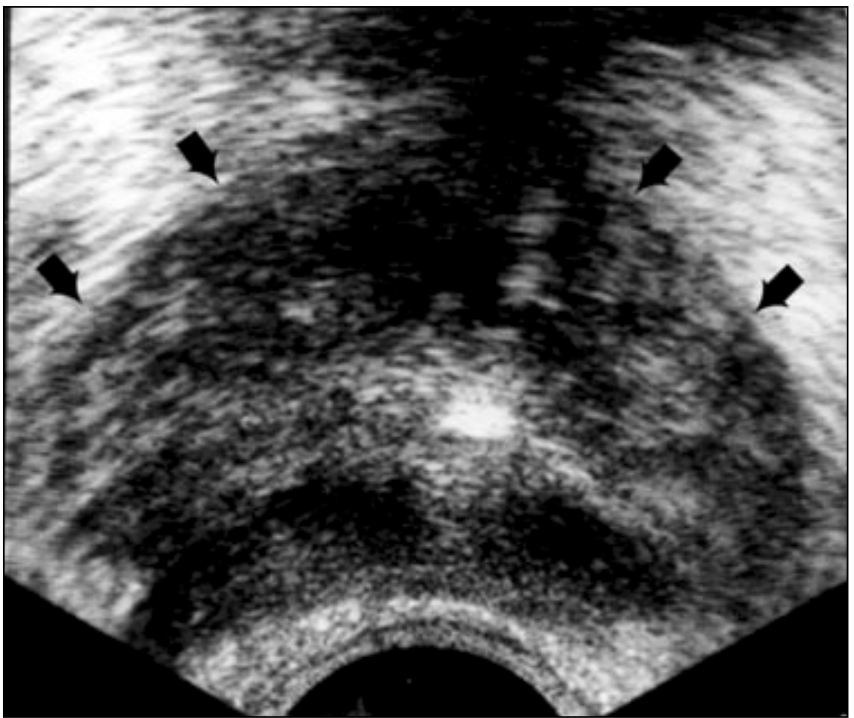

A

Figura 2. Cortes transversal $(\mathbf{A})$ e longitudinais $(\mathbf{B}, \mathbf{C})$ da próstata. Próstata apresentando alteração hipoecogênica difusa, com perda da demarcação entre a zona periférica e a glândula interna. A biópsia revelou tratar-se de adenocarcinoma. Notar a irregularidade dos contornos da próstata (setas).
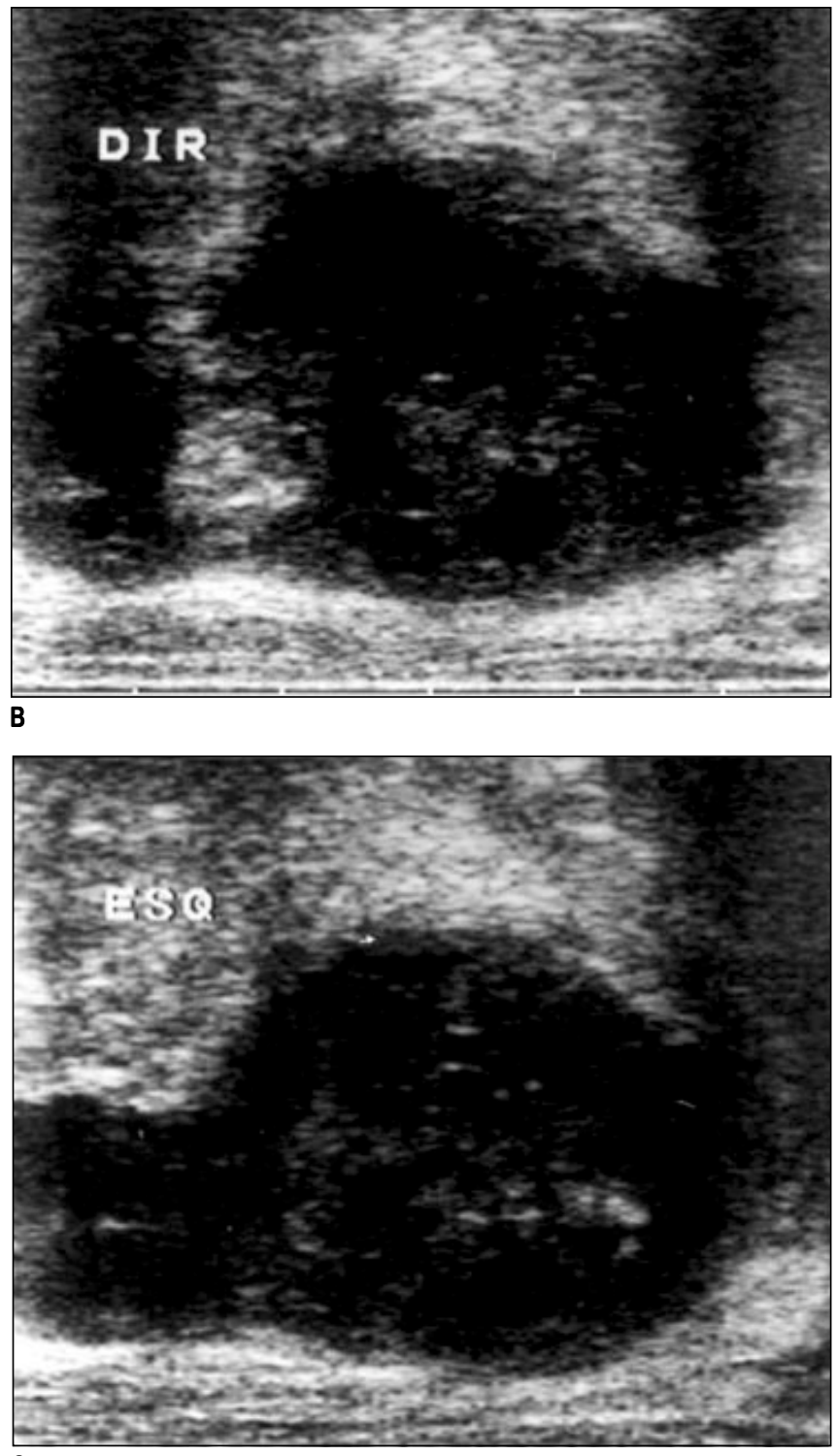

C sido utilizada como modalidade de estadiamento do $\mathrm{CAP}^{(25)}$

Tomando uma direção um pouco diferente dos estudos mais recentes com relação ao CAP, gostaríamos de chamar a atenção para a existência de próstatas que na USER apresentam-se difusamente heterogêneas e hipoecogênicas, com perda da demarcação entre a zona periférica e a glândula interna. Na nossa casuística, a observação deste tipo de alteração constituiu importante critério de suspeita ultra-sonográfica de CAP, já que em 95,4\% das vezes em que a próstata apresentou estas características ultra-sonográficas foi detectado CAP pela biópsia. A associação entre a observação desta alteração ultra-sonográfica e a positividade de CAP na biópsia foi estatisticamente significante.

Há poucas referências na literatura consultada com relação a este tipo de alteração ultra-sonográfica da próstata.

Griffiths et al. ${ }^{(26)}$ referem que a perda de demarcação entre a zona periférica e o restante da glândula pode ser considerada um sinal ultra-sonográfico adicional de CAP, já que $70 \%$ das próstatas dos pacientes por eles estudados que apresentavam CAP extensos, porém confinados, apresentaram estas características na USER.

Para Shinohara et al. ${ }^{(7)}$, a identificação da ausência da margem normal entre a zona periférica e a zona de transição em próstata de aspecto arredondado deve alertar o ultra-sonografista sobre a possibilidade da presença de grandes tumores.

Allen e Embry ${ }^{(27)}$, realizando biópsias de próstatas com alteração difusa da ecogenicidade, encontraram CAP em todas elas.

Não foram encontradas referências mais recentes, na literatura consultada, com relação a esta descrição de alteração ultra-sonográfica suspeita da presença de CAP (alteração hipoecogênica difusa da próstata, caracterizada por perda da demarcação entre a zona periférica e a glândula interna), apesar de que um dos critérios de suspeita ultra-sonográfica de 
CAP, proposto por Leibowitz e Staub(28), foi a perda da arquitetura zonal, que correspondeu a CAP em quatro de quatro casos em que foi observada, mas esta alteração não foi por eles considerada significativa, devido ao pequeno número de pacientes em que foi observada (quatro de 282 pacientes).

Apesar de as descrições das características ultra-sonográficas das próstatas dos trabalhos citados não serem idênticas, elas devem corresponder às mesmas alterações ultra-sonográficas por nós observadas.

\section{CONCLUSÃO}

Concluímos que, apesar de a alteração hipoecogênica difusa da próstata, com perda da demarcação entre a zona periférica e a glândula interna, não constituir apresentação patognomônica do CAP na USER, não podemos deixar de ressaltar a importância desta alteração como critério ultra-sonográfico de suspeita de CAP.

Talvez estudos posteriores analisando casuísticas maiores possam vir a confirmar a importância deste achado ultrasonográfico para o diagnóstico de CAP, definindo melhor a sua especificidade.

\section{REFERENNCIAS}

1. Devonec M, Fendler JP, Monsallier M, et al. Prostatic cancer diagnosis with ultrasonically guided prostatic biopsy: results in 226 cases. In: Therapeutic Progress in Urological Cancers. New York: Alan R. Liss, 1989;35-40.

2. Rifkin MD, McGlynn ET, Choi H. Echogenicity of prostate cancer correlated with histologic grade and stromal fibrosis: endorectal US studies. Radiology 1989;170:549-52.

3. Jansen H, Gallee MP, Schröder FH. Analysis of sonographic pattern in prostatic cancer: comparison of longitudinal and transversal transrectal ultrasound with subsequent radical prostatectomy specimens. Eur Urol 1990;18:174-8.

4. Lee F, Torp-Pedersen S, Littrup PJ, et al. Hypoechoic lesions of the prostate: clinical relevance of tumor size, digital rectal examination, and prostatespecific antigen. Radiology 1989;170(1 Pt 1):29-32.

5. Hernandez AD, Smith JJA Jr. Transrectal ultrasonography for the early detection and staging of prostate cancer. Urol Clin North Am 1990;17:745-57.

6. Shinohara K, Scardino PT, Carter SSC, Wheeler TM. Pathologic basis of the sonographic appearance of the normal and malignant prostate. Urol Clin North Am 1989;16:675-91.

7. Shinohara K, Wheeler TM, Scardino PT. The appearance of prostate cancer on transrectal ultrasonography: correlation of imaging and pathological examinations. J Urol 1989;142:76-82.

8. Melchior SW, Brawer MK. Role of transrectal ultrasound and prostate biopsy. J Clin Ultrasound 1996;24:463-71.

9. Waterhouse RL, Resnick MI. Applications of prostate ultrasonography. Aust N Z J Surg 1991;61:3329.

10. Dyke CH, Toi A, Sweet JM. Value of random USguided transrectal prostate biopsy. Radiology 1990;176:345-9.

11. Sheth S, Hamper UM, Walsh PC, Holtz PM, Epstein JI. Stage A adenocarcinoma of the prostate: transrectal US and sonographic-pathologic correlation. Radiology 1991;179:35-9.

12. el Din KE, de la Rosette JJMCH. Transrectal ultrasonography of the prostate. Br J Urol 1996; 78: 2-9.

13. Kökeny GP, Cerri GG, Cerri LMO, Barros N. Correlations among prostatic biopsy results, transrectal ultrasound findings and PSA levels in diagnosing prostate adenocarcinoma. Eur J Ultrasound 2000; 12:303-13.

14. Hodge KK, McNeal JE, Terris MK, Stamey TA. Random systematic versus directed ultrasound guided transrectal core biopsies of the prostate. J Urol 1989;142:71-5.

15. Hamper UM, Sheth S. Prostate ultrasonography. Semin Roentgenol 1993;28:57-73.
16. Kaye KW, Lightner DJ. Prostate biopsy indices: toward efficient use of transrectal ultrasound. Urology 1993;41:417-20.

17. Pollack HM, Resnick MI. Prostate-specific antigen and screening for prostate cancer: much ado about something? Radiology 1993;189:353-6.

18. Egevad L, Frimmel H, Norberg M, et al. Threedimensional computer reconstruction of prostate cancer from radical prostatectomy specimens: evaluation of the model by core biopsy simulation. Urology 1999;53:192-8.

19. Terris MK. Sensitivity and specificity of sextant biopsies in the detection of prostate cancer: preliminary report. Urology 1999;54:486-9.

20. Veneziano S, Pavlica P, Querzé R, Nanni G, Lalanne MG, Vecchi F. Correlation between prostatespecific antigen and prostate volume, evaluated by transrectal ultrasonography: usefulness in diagnosis of prostate cancer. Eur Urol 1990;18:112-6.

21. Prando A. O valor do Doppler a cores na detecção do câncer da próstata. Radiol Bras 1997;30:23340.

22. Cornud F, Hamida K, Flam T, et al. Endorectal color doppler sonography and endorectal MR imaging features of nonpalpable prostate cancer: correlation with radical prostatectomy findings. AJR 2000;175:1161-8.

23. Halpern EJ, Verkh L, Forsberg F, Gomella LG, Mattrey RF, Goldberg BB. Initial experience with contrast-enchanced sonography of the prostate. AJR 2000;174:1575-80.

24. Prando A, Wallace S. Helical CT of prostate cancer: early clinical experience. AJR 2000;175:3436.

25. Jager GJ, Severens JL, Thornbury JR, de la Rosette JJMCH, Ruijs SH, Barentsz JO. Prostate cancer staging: should MR imaging be used? A decision analytic approach. Radiology 2000;215:445-51.

26. Griffiths GJ, Clements R, Jones DR, Roberts EE, Peeling WB, Evans KT. The ultrasound appearances of prostatic cancer with histological correlation. Clin Radiol 1987;38:219-27.

27. Allen MD, Embry B. Biopsy of the prostate guided by transrectal ultrasonography: early experience in a teaching community hospital. South Med J 1991;84:579-86.

28. Leibowitz CB, Staub PG. Transrectal ultrasoundguided biopsy of the prostate: what you see is not what you get. Australas Radiol 1996;40:240-3. 